\title{
Primary Malignant Melanoma of the Rectum: Long-Term Disease-Free Survival Following Abdomino-Perineal Resection
}

\author{
Emanuel Cavazzoni, Walter Bugiantella, Luigina Graziosi, Emanuele \\ Rosati and Annibale Donini
}

Department of General and Emergency Surgery University of Perugia, Italy

Correspondence should be addressed to: Emanuel Cavazzoni; emanuelcavazzoni@hotmail.com

Received date: 23 February 2014; Accepted date: 23 April 2014; Published date: 25 May 2015

Academic Editor: Fábio Guilherme Campos

Copyright (C) 2015. Emanuel Cavazzoni, Walter Bugiantella, Luigina Graziosi, Emanuele Rosati and Annibale Donini. Distributed under Creative Commons CC-BY 4.0

\begin{abstract}
Objective: Primary Malignant Melanoma (PMM) arising from the digestive, respiratory or genitourinary tract is extremely rare. Rectal PMM accounts for less than $1 \%$ of all melanomas; it is often advanced at initial presentation with a poor prognosis with a 5-year survival below $20 \%$. Treatment of this particular disease is still debated with weak evidence that aggressive surgery may lead to a better outcome. The objective of the present paper is to descibe a case of PMM and review the published literature on the treatment of such extremely rare condition. Methods: A 78 years old man presenting mild anal bleeding underwent a colonoscopy which showed a centimetric mass in the posterior wall of the rectum, less than $1 \mathrm{~cm}$ above the pectinea line. Biopsy revealed amelanotic malignant melanoma infiltrating the submucosa. Preoperative assessment revealed no distant metastases and no nodal involvement. Abdominoperineal resection (APR) with total mesorectal excision and iliac nodes sampling was performed. Results: the patient is alive and disease-free after 48 months from diagnosis. According to a literature review, APR with or without intra abdominal pelvic lymph node dissection represents a more radical approach in patients seeking curative options. However, some series suggest that wide local excision (WLE) may have similar survival outcomes but seems to be burdened with a higher rate of local recurrences. Conclusions: Our experience confirms that treatment recommendations are not standardized and tend to be individualized. An aggressive surgical approach may be justified in patient with a long life expectancy accepting a demolitive surgery and a definitive colonostomy.
\end{abstract}

Keywords: Rectal melanoma; primary; abdomino-perineal resection; wide local excision; survival.

Cite this Article as: Emanuel Cavazzoni, Walter Bugiantella, Luigina Graziosi, Emanuele Rosati and Annibale Donini (2015)," Primary Malignant Melanoma of the Rectum: Long-Term Disease-Free Survival Following Abdomino-Perineal Resection", International Journal of Gastroenterology Research and Practice, Vol. 2015 (2015), Article ID 999601, DOI: 10.5171/2015.999601 


\section{Introduction}

Primary anorectal melanoma is a rare neoplasm, accounting for $24 \%$ of mucosal melanomas, $4 \%$ of all anal tumors and less than $1 \%$ of all melanomas $[1,2]$. The peak of incidence is at the $6^{\text {th }}$ decade [3] with a weak predominance for female [4]. Since the physiopathology of mucosal melanoma is based on the migration of melanocytes from the skin to the mucosal layers, primary malignant melanoma (PMM) of the rectum, located above the anorectal junction, are extremely rare and limited to single case reports. Small primary rectal melanomas are generally asymptomatic, and the lack of early presenting symptoms can be responsible of a delayed diagnosis; uncommonly they can cause mild anal discomfort and bleeding. Due to a high number of advanced stages at diagnosis and an aggressive biological behavior, overall 5-years survival for mucosal melanoma varies between $6-22 \%$, with a mean survival time below 25 months. $[2,4,5,6,7]$. The treatment of primary rectal melanoma is still debated; it can range from local excision to abdomino-perineal resection (APR). Although early studies suggest that aggressive treatment of primary anorectal melanoma with APR is associated with a better prognosis [8, 9], other authors experienced analog survival results with a wide local excision (WLE) of the primary tumor site $[3,7,10,11,12]$.

\section{Case Report}

A 78 years old male presented to our department with an endoscopic diagnosis of rectal neoplasia. The only referred symptom was mild anal bleeding. Tumor's endoscopic appearance was of a flat centimetric polyp of the posterior rectal mucosa, less than $1 \mathrm{~cm}$ above the anorectal junction. Blood tumor markers (CA19.9, CEA) were negative. Biopsy resulted suggestive for a poorly differentiated solid neoplasia; immunochemistry was positive for Melan-A, S-100 protein, HMB-45 and Vimentin suggestive for rectal melanoma. Dermatological, Otolaryngology and Oculist counseling excluded other melanoma localization, and the lesion was then considered a rectal PMM. A CT scan showed no sign of parenchimal or lymphatic metastases while revealing an irregular inspissation of the lower rectum. A total body 18F-FDG PET-CT scan confirmed an isolated pathological activity of the posterior rectal wall with no other sign of captation. Ecoendoscopy showed infiltration of the submucosal layer with no mesorectal lymphadenophaty. The patient underwent abdomino-perineal amputation (Miles' procedure) with iliac lymphnodes dissection and definitive pathology confirmed the presence of a primitive malignant amelanotic melanoma of the lower rectum (Fig.1) focally infiltrating the submucosa with no lymphatic invasion of the mesorectal and internal iliac stations (T2N0M0). Previous byopsic immunochemistry was confirmed, and replication index was 30\% (Ki67-Mib1). Post-operative course was complicated by prolonged ileus resolved with prokinetic drugs administration. The patient did not undergo any adjuvant chemotherapy. Instrumental and clinical follow up including abdominal US and 18F-FDG PET-CT scan was performed every 6 months with no evidence of local or systemic tumor relapse. After 48 months the patient is in good clinical conditions and remains disease free. 


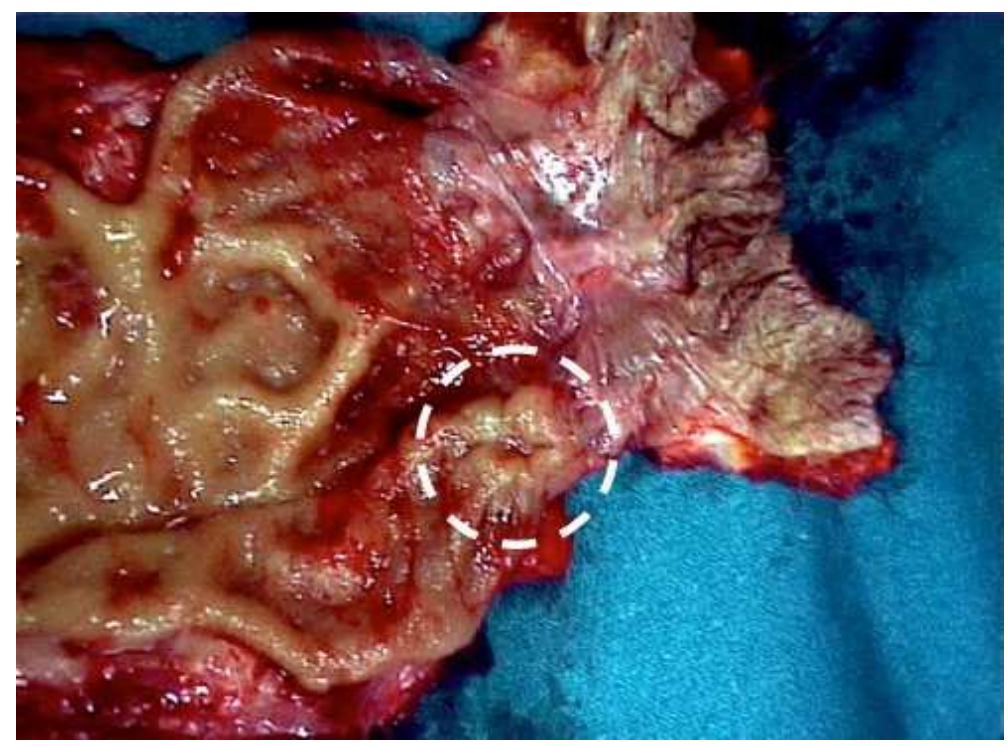

Figure 1: Surgical specimen: primitive malignant amelanotic melanoma of the lower rectum.

\section{Discussion}

The term "rectal" melanoma describes lesions occurring in the rectum at a visible distance from the anorectal junction. The etiopathogenesis of mucosal melanomas is not yet fully elucidated. Distribution of primary mucosal melanomas tends to be strictly close to the mucocutaneous junctions. Melanocytes, originating from the neuroectodermal, are known to migrate to the skin, retina, uveal tract, and other ectodermally derived tissues included the intestinal mucosa. Once there, they may undergo malignant transformation. [13]. Macroscopic appearance of rectal melanoma is of a rilevated lesion or polyp; the majority of patients present a gross pigmented tumour, while only $30 \%$ of patients are diagnosed with an amelanotic melanoma. In such cases the lack of melanin and the uncommon gross appearance may make the endoscopic diagnosis particularly challenging [14]. Unlike cutaneous melanoma, mucosal melanomas do not have precursor lesions and risk factors. As long as the lesions remain in the early stages and do not reach significant dimensions, the most common reported symptom is intermittent anal bleeding. With increased dimensions and depth of infiltration, anal tenesmus, major bleeding and anorectal, pain can be experienced. Lack of specific early symptoms can lead to a late recognition of the disease. Moreover, in precocious stages, differential diagnosis includes benign anorectal pathologies until endoscopy and biopsy are performed. Biopsy proven melanocitic lesion is a landmark in the diagnosis of rectal mucosal melanoma. In case of amelanotic lesions, immunohistochemistry positivity for melanoma associated antigens as Melan-A, S100, HMB-45, Vimentin and Mart-1 can lead to the correct diagnosis, discriminating primary melanoma from other poorly differentiated tumors such as lymphoma or angiosarcoma. Anorectal melanoma prognosis is poor and in general seems to be 
unaffected by the choice of the surgical treatment $[3,7,10,11,12]$. Although the majority of patients are diagnosed in a Stage I disease $(80 \%)$ [15] (No evidence of nodal involvement or distant metastasis), 5-year overall survival remains low (22\%), with a high rate of distant metastasis occurring within two years from diagnosis [6]. Once PMM is diagnosed and staged, there is still controversy regarding the adequate surgical treatment: APR vs. WLE. The rational of APR is based on a wider primary tumour site excision with clear margins and nodal stations removal. Although it is a disabling procedure, it allows a more correct evaluation of the pathological tumor stage and avoids continuous endoscopic survey with an acceptable rate of peri-operative complications. It also represents the better surgical procedure from an oncological point of view. WLE is the other surgical choice. Regardless of the chosen surgical technique (Transanal Endoscopic Microsurgery-TEM vs. simple transanal excision), local excision offers advantages in terms of quality of life and morbidity rate when compared with APR. Given the rarity of the disease, to date there are no controlled trials comparing the two surgical strategies for the treatment of PMM, and the only available data are based on retrospective and historical controls on small groups of patients. A review by Yap et al. [7] summarizes the majority of the studies retrospectively comparing APR vs. WLE. Despite the fact that many reports, including a meta-analyses on 426 patients [10], show no difference in survival between the two surgical strategies, a study from the Memorial Sloan-Kettering Cancer Center on 85 patients [8] found an increased 5-year disease free survival among patients undergoing APR (27\%) compared to those treated by WLE (5\%); in accordance with the rational of a more radical approach to the disease, authors concluded that APR should be reserved for patients with localized PMM and no evidence of nodal involvement. The importance of tumor thickness and nodal metastasis as negative prognostic factors for survival was well illustrated in this study; finally, some cases of long-term survival between patients with nodal disease treated with APR were also reported. Other previous studies confirmed that APR may lead to better control of the disease, reducing the incidence of local recurrence $[10,16,17,18]$. Conflicting findings are described by other study groups, as shown in a recent report of 15 patients affected by anorectal melanoma in which APR seemed to offer no advantage in survival and local relapse when compared to WLE [11]. Authors favoring WLE tend to advocate the usefulness of nodal prophylactic dissection in PMM; however, in our opinion nodal involvement of primary rectal melanoma can be difficult to diagnose preoperatively given the limits of radiological instruments (CT, MRI, endo-US) toward detection of early or micro metastasis. Therefore, only the en-bloc excision of the rectum with the relative mesorectum can lead to a correct diagnosis of stage and nodal tumor clearance, with a possible advantage in terms of recurrence reduction. In addition, most reviews $[7,8,12]$ noted that rectal melanoma tend to diffuse proximally through the submucosal layer; this may account for a greater tendency to involve mesenteric nodes beginning with the mesorectal ones. For this reason, APR represents a logical operative approach in patients with PMM with or without evidence of nodal involvement. Many Authors advocates that size, and in particular depth of the tumour represent the best prognostic index in mucosal melanomas [19]; whereas others suggest that the sole predictive factor for survival is represented by the stage of the disease identified as local, regional or systemic [20]. In both cases, especially for small lesions of the rectal wall, a preoperative assessment of these parameters may be difficult to achieve, especially in regard of the nodal involvement which can be ascertained only after performing a total mesorectal excision. If the aim for surgical therapy of PMM is to find the best compromise between quantity and quality of life, recent reports suggest that in small lesion with a thickness of 1-4 mm, a WLE with a $2 \mathrm{~cm}$ of safe margin would be appropriate [21]. Again, the majority of PMM 
arise close to the anal edge and in strict contact with the anal sphincter, originating several difficulties in respecting safety distances when performing local excision. This could explain the higher rate of local recurrence following WLE as is reported by some Authors [10, 16, 17, 18].. Our decision to perform APR was also based on the patient's acceptance of a terminal colonostomy and his preference for a definitive treatment. In fact, in cases of lesion susceptible of WLE, patients should be aware that local recurrence are common and complications requiring additional surgery may occur, including cases of positive resection margins or the suspicion of lymphnodes involvement at instrumental follow up.

\section{Conclusion}

To date, only few cases of rectal PMM are described in the international literature; this represents the main obstacle in drawing a precise treatment guideline. Therapeutic strategies should then be adjusted considering the prognostic indicators of the disease, which in case of PMM are still undefined. In our experience the choice of a more radical approach leads to a good result in terms of survival, significantly higher if compared to the standard survival rate reported in literature. WLE still have a role in the treatment of rectal melanoma, representing the surgical choice in those patients non eligible for major surgery or in those with advanced disease that could benefit from local palliation. Prospective trials comparing APR and WLE are a major need in helping the choice of the adequate treatment of PMM; given the very low incidence, a meta-analysis on the published case report could be an useful resource to better understand the optimal treatment.

\section{Acknowledgment}

Melissa Meehan RN, University of California at San Diego (UCSD) for grammar review

\section{References}

1. Klas JV, Rothenberger DA, Wong WD, Madoff RD. Malignant tumors of the anal canal: the spectrum of disease, treatment, and outcomes. Cancer. 1999 Apr 15;85(8):1686-93. Review.

2. Chang AE, Karnell LH, Menck HR. The National Cancer Data Base report on cutaneous and noncutaneous melanoma: a summary of 84,836 cases from the past decade. The American College of Surgeons Commission on Cancer and the American Cancer Society. Cancer. 1998 Oct 15;83(8):1664-78.

3. Yeh JJ, Shia J, Hwu WJ, Busam KJ, Paty PB, Guillem JG, Coit DG, Wong WD, Weiser MR. The role of abdomino-perineal resection as surgical therapy for anorectal melanoma. Ann Surg. 2006 Dec;244(6):1012-7.

4. Weinstock MA. Epidemiology and prognosis of anorectal melanoma. Gastroenterology. 1993 Jan;104(1):174-8.

5. Banner WP, Quan P, Woodruff J. Malignant melanoma of the anorectum. Surg Rounds 1982;154:337.

6. Bolivar JC, Harris JW, Branch W, Sherman RT. Melanoma of the anorectal region. Surg Gynecol Obstet. 1982 Mar;154(3):337-41.

7. Yap LB, Neary P. A comparison of wide local excision with abdominoperineal resection in anorectal melanoma. Melanoma Res. 2004 Apr;14(2):147-50.

8. Brady MS, Kavolius JP, Quan SH. Anorectal melanoma. A 64-year experience at Memorial Sloan-Kettering Cancer Center. Dis Colon Rectum. 1995 Feb;38(2):146-51. Review.

9. Wanebo HJ, Woodruff JM, Farr GH, Quan SH. Anorectal melanoma. Cancer. $1981 \mathrm{Apr}$ 1;47(7):1891-900. 
10. Thibault C, Sagar P, Nivatvongs S, Ilstrup DM, Wolff BG. Anorectal melanoma: an incurable disease? Dis Colon Rectum. 1997 Jun;40(6):661-8.

11. Bullard KM, Tuttle TM, Rothenberger DA, Madoff RD, Baxter NN, Finne CO, Spencer MP. Surgical therapy for anorectal melanoma. J Am Coll Surg. 2003 Feb;196(2):206-11.

12. Malik A, Hull TL, Floruta C. What is the best surgical treatment for anorectal melanoma? Int J Colorectal Dis. 2004 Mar;19(2):121-3.

13. Iverson $\mathrm{K}$, Robins RE. Mucosal malignant melanomas. An J Surg 1980;139:660.

14. Tomicic J, Wanebo HJ. Mucosal melanomas. Surg Clin North Am. 2003 Apr;83(2):237-52. Review

15. Patrick RJ, Fenske NA, Messina JL. Primary mucosal melanoma. J Am Acad Dermatol. 2007 May;56(5):828-34. Review.

16. Abbas JS, Karakousis CP, Holyoke ED. Anorectal melanoma: clinical features, recurrence and patient survival. Int Surg. 1980 Sep-Oct;65(5):423-6.

17. Ross M, Pezzi C, Pezzi T, Meurer D, Hickey R, Balch C. Patterns of failure in anorectal melanoma. A guide to surgical therapy. Arch Surg. 1990 Mar;125(3):313-6.

18. Roumen RM. Anorectal melanoma in The Netherlands: a report of 63 patients. Eur J Surg Oncol. 1996 Dec;22(6):598-601.

19. Goldman S, Glimelius B, Påhlman L. Anorectal malignant melanoma in Sweden. Report of 49 patients. Dis Colon Rectum. 1990 Oct;33(10):874-7.

20. Slingluff CL Jr, Vollmer RT, Seigler HF. Anorectal melanoma: clinical characteristics and results of surgical management in twenty-four patients.Surgery. 1990 Jan;107(1):1-9.

21. 21. Weyandt GH, Eggert AO, Houf $M$, Raulf F, Bröcker EB, Becker JC. Anorectal melanoma: surgical management guidelines according to tumour thickness. Br J Cancer. 2003 Dec 1;89(11):2019-22. 